\title{
The effects of staff training on staff confidence and challenging behavior in services for people with autism spectrum disorders.
}

\section{McDonnell, A., Sturmey, P., Oliver, C., Cunningham, S, Hayes, S., Galvin, M., Walshe, C. and Cunningham, C.}

\author{
Cerebra Centre for Neurodevelopmental Disorders, \\ School of Psychology, \\ University of Birmingham
}

Please use this reference when citing this work:

McDonnell, A., Sturmey, P., Oliver, C., Cunningham, S, Hayes, S., Galvin, M., Walshe, C. and Cunningham, C. (2008). The effects of staff training on staff confidence and challenging behavior in services for people with autism spectrum disorders. Research in Autism Spectrum Disorders, 2, 311-319. (DOI:10.1016/j.rasd.2007.08.001).

The Cerebra Centre for Neurodevelopmental Disorders,

School of Psychology, University of Birmingham, Edgbaston, Birmingham, B15 2TT

Website: www.cndd.Bham.ac.uk

E-mail: cndd-enquiries@contacts.bham.ac.uk 


\begin{abstract}
The effects of a 3 day training course in the management of aggressive behaviour in services for people with autism spectrum disorders were investigated using a quasiexperimental design. An experimental group received training over a 10-month period and a contrast group, which had received training before this study, did not. Staff training increased carer confidence, but there were no training effects of measures of staff coping, support or perceived control of challenging behaviours. Staff reports of service user challenging behaviour management difficulties decreased in both the experimental and contrast groups. This study showed that staff training can increase staff confidence in managing aggression in people with autism spectrum disorders.
\end{abstract}


The effects of staff training on staff confidence and challenging behavior in services for people with autism spectrum disorders.

Aggressive behavior in people with mental retardation in community settings is shown by approximately $2-15 \%$ of children and adolescents and approximately $10-15 \%$ of adults (Rojahn \& Tasse, 1996.) Aggression has many negative consequences, including rejection by peers, staff and family members, exclusion from integrated settings, use of restrictive behavioral practices, increased use of psychotropic medications, injuries to self, peers and staff, and increased costs (McDonell \& Sturmey, 1993.) In response to this problem a range of community-based services for aggressive and other challenging behaviors have been developed (Allen, 2001, 2002; Hanson, Weisler, \& Lakin, 2002)

A wide range of interventions have been developed for aggression in people with mental retardation including those based on applied behavior analysis, psychotropic medication and other methods of intervention. A distinction has been made between planned behavioral interventions, such as environmental modifications and skills teaching, and reactive strategies, such as unplanned restraint (Allen, 2001.) There is an extensive literature on planned behavioral intervention (Carr et al., 1999; Didden, Duker \& Cornelius, 1997; Scotti , Ujcich, Weigle, Holland \& Kirk, 1996), but less attention has been paid to evaluation of reactive strategies (Allen, 2001.)

There is some evidence that reactive strategies are widely used in community settings. Emerson (2002) conducted several surveys of children and adults with mental retardation in community services. He surveyed 107 children and adolescents with challenging behaviour who lived in community settings and found that $67 \%$ of them had their challenging behaviour managed 'sometimes' or usually' managed by restraint. $68 \%$ by 
seclusion, and $6 \%$ by sedation. In a second survey of 68 children and adolescents with intellectual disabilities $46 \%$ had experienced restraint, $67 \%$ seclusion, $2 \%$ sedation and $4 \%$ medication over the preceding 6 months. In a third survey of 656 of children with intellectual disabilities, $42 \%$ displayed challenging behavior. Of those who displayed challenging behavior $28 \%$ had experienced physical restraint, $32 \%$ seclusion, $1 \%$ sedation and 3\% mechanical restraint over the preceding six months. Feldman, Atkinson, FotiGervail and Condillac (2004) conducted a similar survey of formal and informal interventions strategies in 625 clients with behavior problems in Ontario from 96 agencies. Ninety-two per cent of the sample lived in community settings and $8 \%$ lived in institutions. They found that $56 \%$ had experienced medication for behavior control, $12.3 \%$ had experienced physical restraint, $11.4 \%$ had experienced confinement time-out, 5.9\% had experienced mechanical restraints and $4.5 \%$ had experienced seclusion. These restrictive procedures were used both in formal programs and informally. Thus, data from both the United Kingdom and Canada indicate that reactive and restrictive strategies are commonly used in community settings.

In response to the need to implement reactive strategies more effectively a staff training courses (Allen, 2001), social policy (Harris, 2002; Harris, Allen, Cornick, Jefferson, \& Mills, 1996)) and regional and state challenging behavioral services (Hanson, et al., 2002) have been developed. Most staff training interventions have been evaluated primarily through non-experimental pre- post-designs (Allen, 2001) although a number of quasi-experimental and experimental studies have been conducted. Allen and Tynan (2000) conducted a quasi-experimental study to evaluate the effects of a staff training course to increase staff members' knowledge of effective management of aggressive behavior and to 
increase their confidence in managing challenging behavior. Their design compared the scores of one group of staff, who had already been trained, with the scores of an untrained group of staff and then also compared the scores of the group of untrained staff prior to training with their scores after training. They found that trained staff knew more and were more confident that untrained staff. After training the scores on knowledge and confidence for the experimental group increased significantly. Other quasi-experimental and experimental studies have found similar benefits of staff training for psychiatric hospital staff (Phillips \& Rudenstam, 1995; Rice, Helzel, Varney \& Quinsey, 1985) and nursing students, although one study found staff training to be ineffective in changing staff (Needham et al., 2004a, 2004b, 2004c.) Finally, Van den Pol, Reid and Fuqua (1983) used a single subject experimental design demonstrated that supervisors could be trained to teach their staff to correctly implement reactive strategies using behavioral skills training. Thus, although there are a number of quasi-experimental and experimental studies demonstrating benefits of staff training, there number is limited and positive outcomes are not always reported. Further, although studies have evaluated staff training procedures to better manage challenging behaviors in adults and children with mental retardation, no studies were identified that worked with services for people with autism spectrum disorders.

McDonell, (1997; McDonnell, Dearden, \& Richens, 1991a, b, c) developed a course to promote the use of simple preventative strategies, a low arousal approach to reducing aggressive behaviours (McDonnell, Johnson, Reeves \& Lane, 1998) and physical interventions, which are designed to avoid pain and which are socially validated (McDonnell, \& Sturmey, 1993, 2000; McDonnell, Sturmey, \& Dearden, 1993). Previous non-experimental studies have demonstrated that it can reduce challenging behaviours and 
the use of restraint for individual clients (McDonell et al., 1998) as well as for entire units (McDonnell \& Reeves, 1996; McDonnell, Walters, Jones, 2002.) Evaluation of 15 courses using a non-experimental pre-post design indicated that staff often gain confidence and knowledge following the course (McDonell \& Sturmey, in press.) Thus, there was a need for a more rigorous evaluation of this staff training course in services for people with autism spectrum disorders. Therefore, we conducted a quasi-experimental evaluation of this three day course for 43 staff who received training and a contrast group of 47 community staff who had previously received training.

\section{Method}

\section{Participants and settings}

There were 90 participants in two service groups. There were 43 participants in the training group. Twenty-eight (65\%) were women and $15(35 \%)$ were men. Ten $(23 \%)$ worked part-time, and 33 (77\%) worked full time. Seven (16\%) had worked for less than one year, $9(21 \%)$ for one to two years, $10(23 \%)$ for 3 to 5 years and $17(40 \%)$ for over five years. There were 47 participants in the comparison group. Twenty-seven (57\%) were women and $20(43 \%)$ were men. Sixteen (34\%) worked part-time, and $31(66 \%)$ worked full time. Eight (17\%) had worked for less than one year, 9 (19\%) for one to two years, 12 $(26 \%)$ for 3 to 5 years and $18(38 \%)$ for over five years.

Both services provided care for adults diagnosed with autistic spectrum disorders.

The staff in the training group provided residential social care and day services to 30 service users in four group homes and a day service. The group homes ranged from a dwelling with four individuals to one house with nine people. This service employed 50 staff. Staff in the comparison group provided residential social care and day services to 48 
service users in six group homes and two-day services. Between six and eight people lived in the group homes.

\section{Staff Training}

All staff in the experimental group participated in the three-day training course (McDonnell, 1997.) Half of this training course involved theoretical components, such as legal issues, causes of aggressive behavior, staff support and low arousal approaches (The remainder of the training course examined high frequency aggressive behaviors, such as hair pulling, biting, grabbing, airway protection, and one form of two person service user chair restraint in an upright posture. Teaching methods for the physical strategies involved modeling methods with rehearsal using role play. Further details of the course content and format can be found in previous publications (McDonnell et al., 1998; McDonnell, et al, 1991a, b, c; McDonnell et al, 1993.)

The three training courses took place over a period of ten months and were conducted by the same trainers. The trainers completed a checklist of course content after each course in order to ensure that the same material was covered on all courses. There was $100 \%$ agreement between the three training course that these items had been delivered.

\section{Measures}

The Staff Support and Satisfaction Questionnaire (3SQ) was a 21-item measure of staff support (Harris \& Rose, 2002). The 3SQ had good test retest reliability $(r=0.82)$ and high levels of internal reliability (Cronbach's alpha $=0.92$, (David, 1997). There were five subscales: role clarity, coping resources, risk factors, supportive people and job satisfaction. Each of the subscales contained items such as ' How clear are you about the main objectives 
you should be working towards in your job?' that were rated on a five-point Likert scale from very clear/very satisfied/always to very unclear/very dissatisfied/never.

The Shortened Ways of Coping Scale (Hatton \& Emerson, 1995) was a 14-item measure with good reliability and internal consistency (average Cronbach's alpha $=0.76$ ). It had 2 subscales: Wishful Thinking (alpha $=0.63)$ and Practical Coping $($ alpha $=0.76) . \quad$ The Wishful Thinking subscale included items such as 'I daydream or imagine a better time or place than the one I am in'. The Practical Coping subscale included items such as 'I think up a couple of different solutions to problems'. Items on both of the scales were rated on a four-point Likert scale from 'not used' to 'used a great deal'.

The Thoughts about Challenging Behavior Questionnaire was a 15-item measure, which examined perceived controllability of staff behavior (Dagnan, 2002). Thoughts that people may have when dealing with a person with a learning disability and challenging behavior (e.g. 'they are trying to wind me up') were rated on a five-point Likert scale from 'agree strongly' to 'disagree strongly'. The internal consistency of the pre-training scores $(N$ $=43)$ was very high $($ alpha $=0.85)$.

The Challenging Behavior Confidence Scale (McDonnell, 1997) was a 15-item measure of self-confidence. Statements about violent people, such as 'I would be able to talk to a potentially violent person,' were rated on an eleven-point Likert scale reflecting how confident participants would be to carry out any of the statements right at this moment. Previous research has produced good internal consistency ratings (Cronbach's Alpha $=$ 0.95; McDonnell, 1997).

The Checklist of Challenging Behavior (Harris, Humphreys \& Thompson, 1994) was a 34-item checklist of various extra-personal challenging behaviors for use with 
individuals with intellectual disabilities. The original checklist contained items, which were collapsed categories "Punching, slapping, pushing or pulling". These items were separated and the new items "Hitting Out (with open hand)", "Punching (clenched fist)" and "Grabbing" were added. Items were rated on the frequency, severity of the injury caused, and the management difficulty that the behavior posed to carers. Ratings were based on behavior over the last three months and ranged from (1) "has not occurred to" (5) "occur very often (daily)". Severity ratings referred to the most serious injury caused by the behavior and ranged from (1) "no injury" to (5) "very serious injury (caused very serious tissue damage such as broken bones, deep lacerations/ wounds requiring hospitalization and/or certified absences from work)." Management difficulty ratings were related to how difficult the carers found the behavior to manage and was scored on a scale of (1) "no problem (I can usually manage this situation with no difficulty at all") to (5) “extreme problem (I simply cannot manage this situation without help.”') Design

A quasi-experimental design was used (Campbell \& Stanley, 1963). An experimental group received training over a ten-month period. This training was compared with a contrast group which had already received the same training system. There were two-time periods for administration of the main measures. The time periods were approximately ten months apart. Thus, if there had been an effect of training the scores on the experimental group, but no the contrast group, should change (Campbell \& Stanley, 1963.). Data were analyzed using two-way ANOVA's and post hoc, Bonferroni corrected $t$ tests.

\section{Procedure}


The measures were completed by a staff who had a good working knowledge of the service users during part of structured interview. The interviewer said 'I am going to read out a list of behaviors and I would like you to respond using the scales provided'. The interviewer read the items out aloud. Staff then rated the items verbally. Wherever possible the same staff completed the measures at time two. The staff measures were administered at both time periods with the following instructions "Please answer the following questions on your own before you talk to other staff about it. Do not put your name on the questionnaire, as the results will be compiled to give group scores. The answers you give will be treated as strictly confidential and only the group scores will be shown to others."

Results

\section{Staff measures}

The relationship between the four staff based measures was investigated using Pearson's product moment correlations. Only one relationship approached significance (3SQ vs. Thoughts about challenging behavior, $r=0.2, \mathrm{p}<.06$ ). The other correlations were all non-significant. Hence, the measures were not inter-correlated.

The mean (and $S D$ ) pre- and post-training confidence scores were: Experimental group / pre 92.1 (34.5), Experimental group / post 111.2 (30.5); Contrast group / pre 87.9 (28.1); and, Contrast group / post 88.0 (27.9). There were significant interaction $(F[1,171]$ $=4.1, p<.05)$ and a main effect of service $(\mathrm{F}[1,171]=9.23, \mathrm{p}<.01)$. Post hoc Bonferroni tests revealed that the contrast group scored significantly lower than the experimental groups at both time 1 and time $2(\mathrm{p}<.05)$. Post hoc Bonferroni corrected $t$ - tests revealed that the only statistically significant differences were between the pre- and post-scores in 
the experimental group $\left(p^{\prime} s<.01\right)$. Thus, staff training had an effect on staff confidence scores.

There were no significant effects of time for the Thoughts about Challenging Behavior Scale $(\mathrm{F}[1,171]=1.37, \mathrm{p}>.05)$ but there was significant main effects of group $(F$ $[1,171]=23.9, p<.001)$. The ways of coping scale divides into two sub-scales 'practical coping' and 'wishful thinking'. The practical coping scale produced no main effect of time $(\mathrm{F}[1,171]=1.07, p>.05)$. There was an effect of group $(\mathrm{F}\{1,171]=7.90, p<.01)$. An analysis of mean scores showed that the comparison group scored lower on this scale at both time 1 and time 2 . The wishful thinking sub-scale produced no significant main effects of group $(\mathrm{F}[1,171]=.571, p>.05)$, or time $(\mathrm{F}[1,171]=.140, p>.05)$.

Staff Support Satisfaction measure produced no main effects of time $(F[1,171]=$ $2.62, \mathrm{p}>.05)$ but there was a significant main effect of group $(F 1,171]=85.7, p<.001)$ which was also found on five subscales [ Role Clarity $(F[1,171]=30.3, \mathrm{p}<.001)$; Coping Resources $(F[1,171]=68.4, p<.001)$; Risk Factors $(F[1,171]=81.9, p<.001)$; Supportive People $(F[1,171]=42.5, p<.001)$; and, Job Satisfaction $(F[1,171]=42.7, p$ $<.001)$. These differences between the mean scores of the experimental and the comparison group merely reflect the naturally occurring differences that existed between the two, groups. There were no other significant effects.

\section{Challenging Behavior Checklist}

There was a main effect of time for management difficulty $(F[1,13]=7.4, p<.02)$, frequency $(F[1,13]=5.6, p<.04)$ and severity $(F[1,13]=13.1, p<.005)$. There were no other significant effects. Both groups reported lower ratings at time 2 . 
This study found that staff training to better manage aggressive behaviors in people with autism spectrum disorders increased staff confidence, but not other measures of staff belief, support or coping. There was no evidence of a reduction in client challenging behaviors as staff in both the treatment and contract group reported significant reductions in challenging behaviors. There was no evidence that training effected staff support (Harris \& Rose, 2002), coping (Hatton \& Emerson, 1995) or perceived control (Dagnan, 2002.) Thus, there was only evidence that training impacted staff confidence.

We observed an effect on staff reports of service user challenging behaviors for both groups. This may be because there truly were no effect of staff training on subjective measures of aggressive behaviors or because the comparison group was an active rather than passive comparison group. In such a situation one might expect the behavior management ratings to decline in both groups. Another possibility is that this measure of behavior may be insensitive or inaccurate. Thus, there may have been effects on client challenging behaviors that would have been detectable through direct observation. Alternatively, it may that in some circumstances that staff training using generic lectures, modeling and role play alone is insufficient to change staff behavior sufficiently to change client challenging behaviour (Cf. Shore, Iwata, Vollmer, Lerman, \& Zarcone, 1995).

The finding that staff training had a specific effect on staff confidence is open to at least two interpretations. First, the course specifically focused on teaching staff strategies to deal with aggressive behavior in a more confident manner and directly focused on staff fears when managing challenging behaviors. Thus, this particular form of staff training may have a relatively specific effect on staff confidence. Earlier research on staff training in 
physical interventions has also found improvements in staff confidence (McDonnell, 1997; Allen \& Tynan, 2000).

The present course teaches staff a strategies that may be helpful with many clients. However, individualization of training in physical interventions may be necessary (Allen, 2001). Perhaps a small number of service users may require highly customized plans for physical intervention whereas a large number of staff and services users may benefit from generic physical intervention training.

Training workshops alone may be necessary but not sufficient for behavioral change to occur (Cullen, 1988.) should also may also need to be followed up in the workplace to increase its effect. Shore et al (1995) demonstrated that a verbal in-service training course explaining procedures to direct care staff was ineffective at changing staff behaviors related to implementing behavioral interventions and client challenging behaviors. Thus, it may be important to supplement staff training workshops, such as the one evaluated here, by ongoing consultation and further staff training and support which involves practicing skills with the client under supervision and whilst receiving feedback on implementation.

There were a number of limitations to this study. The use of a quasi-experimental design may limit the confidence with which we can conclude that training caused a change in staff confidence (Campbell \& Stanley, 1963.) Future research should also focus on variables that mediate staff confidence, such as staff fear and anger may be useful to consider in future studies. The effect of training on staff behavior measured through direct observation should also be addressed. 


\section{References}

Allen, D. (2000) Recent research on physical aggression in persons with intellectual disability: An overview. Journal of Intellectual and Developmental Disability, 25, 41-57.

Allen, D. (Ed., 2001) Training carers in physical interventions. Research towards evidence-based practice. BILD: Kidderminster.

Allen D. \& Tynan H (2000.) Responding to aggressive behavior: impact of training on staff members' knowledge and confidence. Mental Retardation, 38, 97104.

Campbell, D. \& J. Stanley. (1963). Experimental and Quasi-Experimental Designs. Chicago: Rand McNally.

Carr, E. G., Horner, R. H., Turnbull, A. P., Marquis, J. G., McLaughlin, D. M., McAtee, M. L.,Smith, C. E., Ryan, K. A.,Ruef, M. B., Doolabh, A. Baddock, D. (1999). Positive Behavior Support for People with Developmental Disabilities: A Research Synthesis. Washington, DC: American Association on Mental Retardation.

Cullen, C. (1988.) Review of staff training: The emperors old clothes. The Irish journal of Psychology, 9, 309-323.

Dagnan, D, (2002.) Personal communication.

Didden, R., Duker, P.C. \& Korzilius, H. (1997) Meta-analytic study on treatment effectiveness for problem behaviors with individuals who have mental retardation. American Journal on Mental Retardation, 101, 4, 387-399. 
Emerson, E. (2002.) The prevalence of use of reactive management strategies in community-based services in the UK. In: D. Allen, (Ed. ) Ethical approaches to physical interventions. Responding to challenging behavior in people with intellectual disabilities, (pp15-30.) Plymstock: BILD Publiscations..

Feldman, M. A., Atkinson, L., Foti-Gervais, L. \& Condillac, R. (2004.) Formal versus informal interventions for challenging behaviour in persons with intellectual disabilities. Journal of Intellectual Disabilities Research, 48, 60-68.

Hanson, R. H., Weisler, N. A., \& Lakin, K. C. (2002.) Crisis prevention and response in the community. Washington, DC: American Association on Mental Retardation.

Harris, J. (2002). Training on physical interventions: Making sense of the market. In: D. Allen, (Ed. ) Ethical approaches to physical interventions. Responding to Challenging behavior in people with intellectual disabilitie, (pp. 134-152.) Plymstock: BILD Publications.

Harris, J., Allen, D., Cornick, M., Jefferson, A. \& Mills, R. (1996) Physical interventions. a policy framework. BILD/NAS: Kidderminster.

Harris P., Humphreys J. \& Thomson G. (1994). A checklist of challenging behaviour: The development of a survey instrument. Mental Handicap Research, 7, 118-133.

Harris, P. \& Rose, J. (2002) Measuring staff support in services for people with intellectual disability: the Staff Support and Satisfaction Questionnaire. Journal of Intellectual Disability Research, 46, 2, 151-157. 
Hatton, C. \& Emerson, E. (1995.) The development of a Shortened Ways of Coping Scale for use with direct care staff in learning disabilities services. Mental Handicap Research, 8, 237-251.

McDonnell, A. (1997) Training care staff to manage challenging behaviour: An evaluation of a three-day course. British Journal of Developmental Disabilities, 43, 156-161.

McDonnell, A., Dearden, B. \& Richens, A. (1991a) Staff training in the management of violence and aggression: 1- Setting up a training system. Mental Handicap, 19, 7376.

McDonnell, A., Dearden, B. \& Richens, A. (1991b) Staff training in the management of violence and aggression: 2- Avoidance and Escape Principles. Mental Handicap, 19, 109-112.

McDonnell, A., Dearden, B. \& Richens, A. (1991c) Staff training in the management of violence and aggression: 3- Physical Restraint. Mental Handicap, 19, 151-154.

McDonnell A.A. \& Reeves S. (1996). The adoption of a non seclusion policy on a locked ward for people with a learning disability: A description of the process. Nursing Times, 92, 42-44.

McDonnell, A. \& Sturmey, P. (1993) Managing violent and aggressive behaviours of people with learning difficulties. In Jones, R.S.P. \& Eayrs, C. (Eds) Challenging Behaviours and Mental Handicap: A Psychological perspective. Kidderminster: BILD.

McDonnell, A. \& Sturmey, P. (2000). The social validation of three physical restraint procedures: a comparison of young people and professional groups. Research in Developmental Disabilities, 21, 85-92. 
McDonell, A. A. \& Sturmey, P. (in press.) Evaluation of a staff training course to manage violent and aggressive behaviour. Journal of Applied Research in Intellectual Disabilities.

McDonnell, A., Sturmey, P. \& Dearden, B. (1993) The acceptability of physical restraint procedures for people with a learning difficulty. Behavioural and Cognitive Psychotherapy, 21, 225-264.

McDonell, A. A., Waters, T. \& Jones, D. (2002). Low arousal approaches in the management of challenging behavior. In Allen, D. (2001) Training carers in physical interventions. Research towards evidence-based practice, (pp. 104-113.) BILD: Kidderminster.

Needham, I., Abderhalden, C., Zeller, A., Dassen, T., Haug, H.J., Fischer, J.E. \& Halfens, R.J.G. (in press a). the effect of a training course in aggression Management on nursing students' confidence and perception of aggression. Journal of Nursing Education

Needham, I., Abderhalden,C., Halfens,R.J.G., Dassen, T., Haug, H.J., \& Fischer J.E. (in press b) The effect of a training course in aggression management on mental health nurses' perception of aggression: A randomised controlled trial. Journal of Psychiatric and Mental Health Nursing.

Needham, I., Abderhalden,C., Meer, R., Dassen, T., Haug, H.J., Halfens, R.J.G. \& Fischer, J.E. (in press c) The effectiveness of two interventions in the management of patient violence in acute mental inpatient settings: Report on a pilot study. Journal of Psychiatric and Mental Health Nursing. 
Phillips, D., \& Rudestam, K.E. (1995). The effect of non-violent self defence training on male psychiatric staff members: aggression and fear. Psychiatric Services, 43, 164 $-168$.

Rice, M.E., Helzel, M.F., Varney, D.W. \& Quinsey, V.L. (1985). Crisis Prevention and Intervention training for psychiatric hospital staff. American Journal of Community Psychology, 13, $289-304$.

Rojahn, J., \& Tassé, M. J. (1996). Psychopathology in mental retardation. In J. W. Jacobson, J. A. Mulick (Eds.), Manual on mental retardation and professional practice (pp. 147-156). Washington, D.C.: American Psychological Association.

Scotti, J. R., Ujcich, K. J., Weigle, K .L., Holland, C. M., \& Kirk, K. S. (1996) Interventions with challenging behavior of persons with developmental disabilities: A review of current research practices. Journal of the Association of Persons with Severe Handicaps, 21, 123-134.

Shore, B. A., Iwata, B. A., Vollmer, T. R., Lerman, D. C., \& Zarcone, J. R. (1995). Pyramidal staff training in the extension of treatment for severe behavior disorders. Journal of Applied Behavior Analysis, 28, 323-332.

Van den Pol, R. A., Reid, D. H., \& Fuqua, R. W. (1983). Peer training of safety-related skills to institutional staff: Benefits for trainers and trainees. Journal of Applied Behavior Analysis, 16, 139-156. 